\title{
The subjective and objective very long-term outcomes of TVT in the COVID era: A 20-year follow-up
}

\author{
Andrea Braga ${ }^{1,2}$ - Giorgio Caccia ${ }^{1} \cdot$ Andrea Papadia $^{2,3} \cdot$ Fabiana Castronovo $^{1} \cdot$ Stefano Salvatore ${ }^{4}$. \\ Chiara Scancarello ${ }^{5} \cdot$ Marco Torella $^{6} \cdot$ Fabio Ghezzi $^{5} \cdot$ Maurizio Serati $^{5}$
}

Received: 15 July 2021 / Accepted: 6 January 2022 / Published online: 1 March 2022

(c) The Author(s) 2022

\begin{abstract}
Introduction and hypothesis Few studies in literature have assessed the long-term durability and mesh-related complications of mid-urethral slings (MUSs). The aim of this study is to assess the efficacy and safety of retro-pubic tension-free vaginal tape (TVT) 20 years after implantation for the treatment of female stress urinary incontinence (SUI).

Methods A prospective observational study was conducted in two urogynaecologic units in two countries. All the patients involved were consecutive women with urodynamically proven pure SUI treated by TVT. The patients underwent preoperative clinical and urodynamic evaluations. Subjective outcomes, objective outcomes and adverse events were recorded during the follow-up period.

Results Fifty-two patients underwent a TVT surgical procedure. Twenty years after surgery, 32 out of 36 patients $(88.8 \%)$ declared themselves cured $(p=0.98)$. Similarly, 33 out of these 36 patients $(91.7 \%)$ were objectively cured $(p=0.98)$. No significant deterioration of subjective and objective cure rates was observed over time ( $p$ for trend 0.50 and 0.48 ). Fifteen of the 36 patients $(41.6 \%)$ at the 20 -year follow-up reported the onset of de novo overactive bladder (OAB) $(p=0.004)$. No significant vaginal bladder or urethral erosion or de novo dyspareunia was recorded and no patient required tape release or resection during this period. The cause of death of seven out of ten women who died in the last year of the follow-up period was coronavirus disease 19 (COVID 19).

Conclusions The 20-year results of this study showed that TVT is a highly effective and safe option for the treatment of SUI. The impact of COVID 19 on the mortality rate of elderly women has drastically reduced the number of eligible patients for future evaluations in our region.
\end{abstract}

Keywords COVID $\cdot$ Long-term follow-up $\cdot$ Mid-urethral sling $\cdot$ Stress urinary incontinence, TVT

Andrea Braga

andrea.braga@eoc.ch; andybraga@libero.it

1 Department of Obstetrics and Gynaecology, EOC - Beata Vergine Hospital, Mendrisio, Switzerland

2 Faculty of Biomedical Sciences, Università della Svizzera Italiana, Lugano, Switzerland

3 Department of Obstetrics and Gynaecology, EOC - Civico Hospital, Lugano, Switzerland

4 Department of Obstetrics and Gynaecology, IRCCS San Raffaele Scientific Institute, Milan, Italy

5 Department of Obstetrics and Gynaecology, University of Insubria, Varese, Italy

6 Department of Gyanecology, Obstetric and Reproductive Science, Second University of Naples, Naples, Italy

\section{Introduction}

Since their introduction [1], tension-free vaginal tape midurethral slings (Gynecare TVT System ${ }^{\circledR}$ ) have been considered the most effective and safest procedure for the treatment of female stress urinary incontinence [2-4]. However, in recent years, the criticisms regarding the use of transvaginal mesh for pelvic organ prolapse (POP) repair have also involved the mesh intended for the treatment of SUI.

Although the notice issued by the United States Food and Drug Administration (US FDA) considered MUSs to be relatively safe [5], in July 2018 the British government announced that the use of mesh for the treatment of POP and urinary incontinence (UI) was to be paused. Conversely, leading urological and urogynaecological associations have stated the importance of distinguishing between the mesh 
used for POP and the one used for SUI [6]. In a review of statements on the use of synthetic mesh for POP and SUI, the Urogynaecology and Pelvic Floor Committee within the International Federation of Gynaecology and Obstetrics (FIGO) drew the conclusion that there is strong evidence to support the use of synthetic mesh MUSs in the treatment of SUI [7]. In fact, in short- and medium-term follow-ups, a large amount of published data has confirmed the similar efficacy but less morbidity of synthetic slings compared with conventional non-mesh sling techniques [8,9]. Only few studies in literature have assessed the long-term durability and the mesh-related complications of MUSs, producing results that are extremely encouraging $[2,10,11]$. Nevertheless, there is not enough evidence available on the longlasting benefits of MUS procedures to be able to recommend these techniques with a robust level of evidence.

Ideally, randomized or prospective clinical trials with a long-term follow-up would be the optimal method for evaluating sling procedures. However, this type of study represents a great challenge for researchers due to many factors that could influence the results, such as cost, the time required and the bias that may occur, especially if there is a significant loss of subjects during the follow-up period (withdrawal by the subjects or death). The latter point is crucial for the long-time evaluation of any surgical procedure. Unfortunately, in the last year, many countries have reported an increased mortality rate due to the novel severe acute respiratory syndrome coronavirus 2 (SARS-CoV-2).

The aim of the present study is to evaluate the long-term efficacy and safety of a TVT procedure in women who underwent a minimum 20-year follow-up.

\section{Materials and methods}

This is an update of a previous prospective study performed in two urogynaecologic units, namely the EOC-Beata Vergine Hospital, Mendrisio, Switzerland, and the University of Insubria, Varese, Italy. As reported in the previous study [2], we enrolled all consecutive women who complained of pure SUI symptoms with urodynamically proven USI between January 1998 and January 2000. All patients deemed eligible for surgical treatment were scheduled for a TVT procedure (Gynecare TVT System ${ }^{\circledR}$; Ethicon Inc., Somerville, NJ, USA). Women with a previous history of radical pelvic surgery, psychiatric and neurologic disorders, concomitant vaginal prolapse $>$ stage 1 according to the Pelvic Organ Prolapse Quantification (POP-Q) system [12], OAB symptoms, urodynamically proven $\mathrm{DO}$ and postvoid residual $>100 \mathrm{ml}$ were excluded from the study. The preoperative evaluation included the patient's medical history, a physical examination, a voiding diary, urinalysis and complete urodynamic testing. A physical examination was performed with the patient in the lithotomy position and POP was described during a maximal Valsalva manouevre according to the POP-Q system [12]. All the women were evaluated with urodynamic studies as previously described [13] [including uroflowmetry, filling cystometry, Valsalva leak-point pressure (VLPP) measurement and a pressure/ flow study] by a trained urogynaecologist, using a standardized protocol in accordance with the best urodynamic practice guidelines of the International Continence Society [14]. Urethral hypermobility was defined by a Q-tip test $>$ $30^{\circ}$. Patients were included regardless of their Q-tip test and VLPP values. All methods, definitions and units were updated in agreement with the latest version of the International Continence Society standardization of terminology [15]. All patients also completed the International Consultation on Incontinence Questionnaire-Short Form ICIQ-SF [16]. All the TVT procedures were performed according to the technique originally described by Ulmsten [1]. General or spinal anaesthesia was used in accordance with anaesthesiological requirements and/or the patient's preference, as previously reported [17]. Postoperative evaluations were mandatory at 1, 5, 10, 15, 17 and 20 years in the two centres and intermediate visits were scheduled at the physician's discretion. The standard of care follow-up in our clinics is that all subjects are asked to complete the following questionnaires and undergo the physical examination components listed in the follow-up evaluation. Every follow-up visit included the patient's medical history, a physical examination, a voiding diary, a stress test and an evaluation of subjective satisfaction [18]. A stress test was performed in the lithotomy and upright positions with a full bladder (ultrasonographic measurement $>400 \mathrm{ml}$ ). "Objective cure" was defined as the absence of urine leakage during the stress test. To define the subjective outcomes at 1, 5, 10, 15, 17 and 20 years, all patients completed the ICIQ-SF, the PGI-I scale (a 7-point scale, with a range of responses from 1, "very much improved", through to 7, "very much worse") [19] and a patient-satisfaction scale (a single, self-answered, Likert-type scale of $0-10$ that grades the patient's degree of satisfaction regarding continence, where 0 represents "not satisfied," and 10, "satisfied") [20]. Subjective success was indicated by both "very much improved" or "much improved" (PGI-I $\leq 2$ ) and a patient-satisfaction score $\geq 8$, as previously described in 2011 by Abdel-Fattah et al. [21]. Additional UDSs were also performed when women complained of the onset of de novo overactive bladder (OAB) symptoms. The Declaration of Helsinki was followed, and preoperative written informed consent for TVT implantation was obtained from all the patients in this observational prospective evaluation. The study does not require ethics/ institutional review board approval because normal clinical practice has been followed [22] 


\section{Statistical analysis}

Statistical analysis was performed with IBM-SPSS v.17 for Windows (IBM Corp, Armonk, NY, USA). Continuous variables were reported as the median and interquartile range. We used the $\chi^{2}$ test and $\chi^{2}$ test for trend to analyse and compare the surgical outcomes during follow-up. The $\chi^{2}$ test for trend can better assess if the success of the surgical procedure tends to decrease over time by comparing the cure rates at the different follow-up visits $(1,5,10,15,17$ and 20 years). The null hypothesis is that there is no association between the cure rate of TVT and the time. The one-way analysis of variance was used to compare continuous series of variables in the comparison of the scores used to measure the subjective outcomes. The Cox proportional hazards model was used for univariate analysis to evaluate factors potentially affecting the risk of recurrence (subjective or objective) during the study period. Statistical significance was considered achieved when $p<0.05$. The analysis of success data was performed by plotting by Kaplan-Meier survival curves, which were compared using the long-rank (Mantel-Cox) test.

\section{Results}

Fifty-two consecutive women with proven USI, who fulfilled the inclusion criteria, i.e., had undergone a TVT procedure, were considered. At the 20-year follow-up, 36 (69.2\%) patients were available for evaluation. The baseline patient characteristics of these 36 patients are reported in Table 1 .

Table 1 Baseline patients characteristics

\begin{tabular}{ll}
\hline Characteristics & $n=52$ \\
\hline Age, years, median, (IQR) & $60(51-72)$ \\
BMI, kg/m², median, (IQR) & $25.9(25-28)$ \\
Obese, BMI $\geq 30$, no. (\%) & $6(11.5)$ \\
Sexually active, no. (\%) & $40(77)$ \\
Menopausal, no. (\%) & $43(82.3)$ \\
HRT, no. (\%) & $17(32.7)$ \\
Recurrent UTI, no. (\%) & $0(0)$ \\
Previous vaginal deliveries, median, (IQR) & $2(1-4)$ \\
Macrosome ( $\geq 4000$ g), no. (\%) & $5(9.6)$ \\
Operative delivery (vacuum/forceps), no. (\%) & $4(15.4)$ \\
Previous hysterectomy, no. (\%) & $12(46.1)$ \\
Urethral hypermobility, no. (\%) & $44(84.6)$ \\
VLPP <60 cmH ${ }_{2} \mathrm{O}$, no. $(\%)$ & $28(53.8)$ \\
ICQ-SF preop. & $17(16-17)$ \\
\hline
\end{tabular}

IQR: interquartile range; BMI: body mass index

HRT: hormone replacement therapy; UTI: urinary tract infection
At the 20-year follow-up, six patients (11.5\%) were lost to follow-up or could no longer be evaluated, while ten (19.2\%) had died from medical causes unrelated to the TVT procedure ( 2 after the 10-year examination, 1 after the 15-year examination and 7 after the 17-year examination).

Figure 1 displays the flowchart of the study. All the women lost to follow-up were subjectively cured at their last follow-up visit. Subjective and objective cure rates are summarized in Table 2 and displayed in Fig. 2.

Twenty years after the TVT procedure, 32 out of the $36(88.8 \%)$ available patients were subjectively cured ( $p$ for trend 0.50$)$, whereas 33 out of the $36(91.7 \%)$ patients were objectively cured ( $p$ for trend 0.48 ). In addition, at the 20-year follow-up, we observed a significant trend of de novo overactive bladder occurrence in 15 out of the 36 patients [ $(41.6 \%)(p=0.004)]$. The long-term subjective outcomes, reported in Table 3, showed no significant deterioration over time.

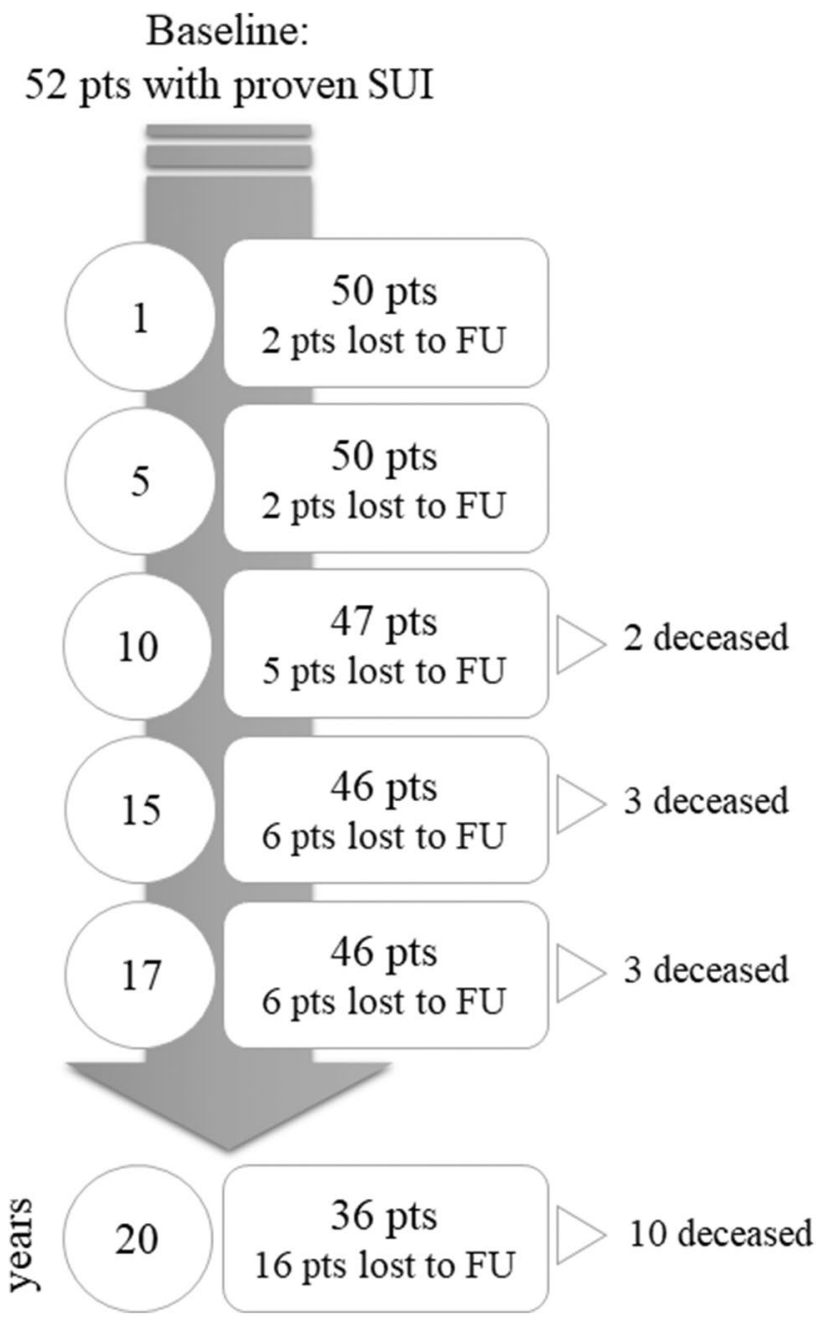

Fig. 1 Flow chart TVT 20 years 
Table 2 Cure rates at the 1-, 5-, 10-, 15-, 17- and 20-year follow-up visits

\begin{tabular}{|c|c|c|c|c|c|c|c|}
\hline & $1 \mathrm{yr}$ & $5 \mathrm{yr}$ & $10 \mathrm{yr}$ & $15 \mathrm{yr}$ & $17 \mathrm{yr}$ & $20 \mathrm{yr}$ & $p$ value \\
\hline $\begin{array}{l}\text { Subjective outcomes } \\
\text { Satisfied (N) }\end{array}$ & $92 \%(46 / 50)$ & $92 \%(46 / 50)$ & $89.3 \%(42 / 47)$ & $89.1 \%(41 / 46)$ & $89.1 \%(41 / 46)$ & $88.8 \%(32 / 36)$ & $0.98^{\mathrm{a}} 0.50^{\mathrm{b}}$ \\
\hline $\begin{array}{l}\text { Objectively cured } \\
\text { Objectively cured (at stress test) }\end{array}$ & $94 \%(47 / 50)$ & $94 \%(47 / 50)$ & $93.6 \%(44 / 47)$ & $91.3 \%(42 / 46)$ & $91.3 \%(42 / 46)$ & $91.7 \%(33 / 36)$ & $0.98^{\mathrm{a}} 0.48^{\mathrm{b}}$ \\
\hline $\begin{array}{l}\text { De novo overactive bladder } \\
\text { Onset of OAB }\end{array}$ & $12 \%(6 / 50)$ & $12 \%(6 / 50)$ & $19.1 \%(9 / 47)$ & $23.9 \%(11 / 46)$ & $32.6 \%(15 / 46)$ & $41.6 \%(15 / 36)$ & $0.004^{\mathrm{a}}$ \\
\hline
\end{tabular}

${ }^{\mathrm{a}}$ Chi-square test; ${ }^{\mathrm{b}}$ chi square test for trend

The null hypothesis is that there is no association between the cure rate of TVT and the time

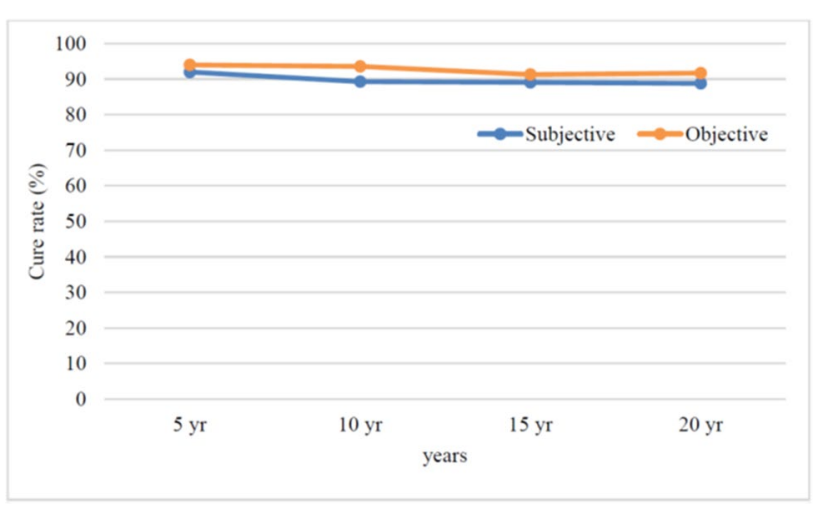

Fig. 2 Recurrence-free (disease-specific) survival curve of women undergoing TVT

The Clavien-Dindo classification of long-term complications is reported in Table 4. No significant vaginal bladder or urethral erosion or de novo dyspareunia was registered in our study population, and no patient required tape release or resection during these 20 years. The univariate analysis did not find any risk factor statistically associated with the recurrence of SUI.

Interestingly, the cause of death of seven out of the ten women who died in the last year of the follow-up period was COVID 19 (Fig. 3 and Table 5).
Table 4 Clavien-Dindo classification of long-term complications

\begin{tabular}{lll}
\hline Complication & $N=36$ & Action \\
\hline $\begin{array}{l}\text { Clavien I } \\
\begin{array}{c}\text { Persistence of voiding } \\
\text { dysfunction }\end{array}\end{array}$ & $2(3.8 \%)$ & Observation \\
$\begin{array}{c}\text { Clavien II } \\
\text { De novo OAB }\end{array}$ & $15(41.6 \%)$ & $\begin{array}{c}\text { Antimuscarinics/ } \\
\beta \text {-agonists }\end{array}$ \\
Recurrent UTIS & $2(3.8 \%)$ & $\begin{array}{c}\text { Antimicrobial } \\
\text { prophylaxis or } \\
\text { therapy }\end{array}$ \\
\hline
\end{tabular}

Data are expressed as an absolute number (\%)

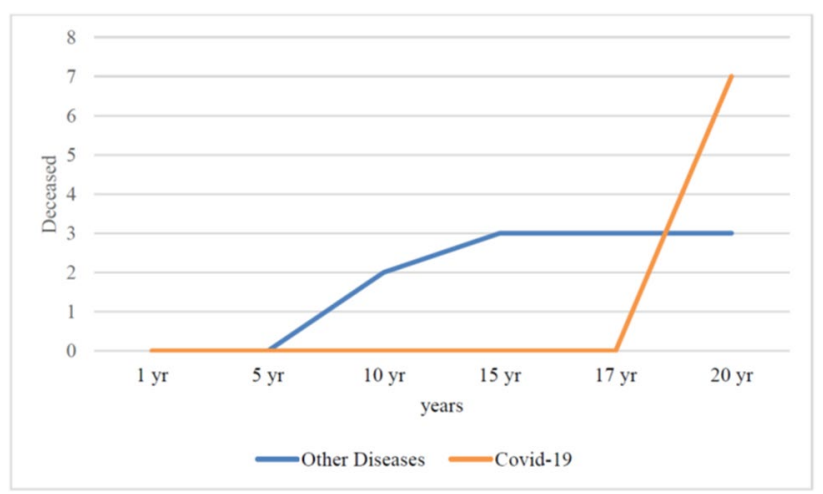

Fig. 3 Patients who died during the follow-up period

Table 3 Subjective outcome scores over time after TVT

\begin{tabular}{llllllll}
\hline & Baseline & 1 year & 5 years & 10 years & 15 years & 17 years & 20 years \\
\hline ICIQ-sf & $17(16-17)$ & $0(0-8)$ & $0(0-10)$ & $0(0-10)$ & $0(0-12)$ & $0(0-12)$ & $0(0-12)$ \\
"Very much better" or & & $46 / 50$ & & $42 / 47$ & $41 / 46$ & $41 / 46$ & $32 / 36$ \\
"much better" on PGI-I & & $(92 \%)$ & & $(89.3 \%)$ & $89.1 \%$ & $89.1 \%$ & $88.8 \%$ \\
Patient Satisfaction Scale & & & $10(8-10)$ & & $10(8-10)$ & $10(8-10)$ & $10(7-10)$ \\
\hline
\end{tabular}

Data are expressed as an absolute number (\%) or median (interquartile range)

*One-way analysis of variance (ANOVA) 
Table 5 Rate of patients who died and median age

\begin{tabular}{llllllll}
\hline & 1 year & 5 years & 10 years & 15 years & 17 years & 20 years & $p$ value \\
\hline Patients deceased & - & - & 2 & 3 & 3 & 10 & $0.006^{\text {a }}$ \\
\multirow{2}{*}{ Median age of pts deceased } & - & - & $(4.2 \%)$ & $(6.5 \%)$ & $(6.5 \%)$ & $(27.7 \%)$ & \\
\hline
\end{tabular}

Data are expressed as an absolute number (\%) or median (interquartile range)

${ }^{a}$ Chi-square test

*One-way analysis of variance (ANOVA)

\section{Discussion}

The present study, which is the first in the literature to the best of our knowledge, shows the subjective and objective outcomes of the TVT procedures undergone by women with pure stress incontinence 20 years after surgery. We found that TVT is a highly effective and safe procedure, with very long-lasting efficacy.

Following the notice issued by the US FDA in 2011 and the recent announcement of the British government, the use of MUSs for female SUI has come under scrutiny because of the reports of severe vaginal mesh complications. However, all these restrictions failed to make the distinction between mesh for incontinence and mesh for prolapse. The European Commission's Scientific Committee on Emerging and Newly Identified Health Risks (SCENIHR) [23] stated that morbidity related to use of prosthetic transvaginal mesh is greater in POP than in SUI treatment.

In fact, as shown by the results of a large populationbased cohort study [24], MUS procedures have a lower risk of immediate (adjusted relative risk [aRR] 0.44 [95\% CI 0.36-0.55]) and later complications (1.12 [0.98-1.27]) (all ratios are for retropubic mesh) than POP procedures. In particular, compared with non-mesh repair, the mesh repair of anterior compartment prolapse was associated with a significantly increased risk of later complications [3.15 (2.46-4.04)]. This study, which is included in the Scottish Independent Review [25] on the use of transvaginal mesh implants in the treatment of female SUI, supports the use of mesh procedures for incontinence, although further research on longer term outcomes would be beneficial. Moreover, an updated meta-analysis by Fusco et al. [8] confirms the superiority of MUSs over Burch colposuspension in terms of overall and objective continence rates as well as equivalent overall and subjective continence rates regarding pubovaginal slings, although MUSs present a statistically significantly lower incidence of storage lower urinary tract symptoms (LUTS).

Despite these advantages and the low complication rate of MUSs [26], the occurrences of mesh erosion and pain have recently become a major concern. The Cochrane Review Collaboration on the long-term outcomes of MUSs reported low incidence rates of vaginal tape erosion $(0.4 \%-1.5 \%)$ and groin pain $(0.4 \%-1.6 \%)$ [27]. Another recent study on the long-term safety of sling mesh implants for SUI reported a risk of mesh erosion of $3.7 \%$ with a $6.7 \%$ reoperation rate at the 7-year follow-up [28]. Nevertheless, the growing international controversy on the use of vaginal mesh has led to these surgical techniques being reconsidered and it has highlighted the need for more information on the longterm results and adverse effects of the mesh used to treat SUI [29]. Unfortunately, two problems arise in this regard:

(1) Although the data are encouraging, only two studies in literature have evaluated the long-term outcomes of TVT with a follow-up of at least 17 years. Nilsson et al. [10] reported an objective cure rate of $91.3 \%$, assessed by a cough stress test, and an $87.2 \%$ subjective cure rate, evaluated by PGI-I, as well as by some specifically validated questionnaires. However, only $51.1 \%$ of the women were assessed with an objective examination, and no UDS evaluation was performed, while in our previous evaluation of the present series [2], 17 years after surgery $89.1 \%$ of the women declared themselves cured and $91.4 \%$ were objectively cured.

(2) The COVID 19 pandemic may represent a significant hurdle for future research on the long-term efficacy and safety of MUSs, especially in regions that have experienced a similar mortality rate to ours.

If it is considered that the mean age of patients dying from SARS-CoV-2 infection is 81 (median 83, range 0-109, IQR 75-88) years and $49.3 \%$ of these are women [11], this could limit the long-term evaluation of this technique, especially considering that the median age of patients who underwent a TVT procedure 20 years ago was around 60 years old $[2,3,10]$. This means that we may not have sufficient data available in the long term for an adequate evaluation of this procedure. In the present study we have recorded a great increase in mortality in the 3 years since the last follow-up due to SARS-CoV-2 infection (7/10 overall deaths) (Fig. 3).

It is interesting to note that no late mesh-related complications appeared during the 20-year follow-up period. However, as in the previous study, we recorded a statistically 
significant growing trend of de novo $\mathrm{OAB}$, which increased from $32.6 \%$ to $41.8 \%$ in the last 3-year postoperative period.

This observation could reflect the ageing of the patients rather than being a direct consequence of surgery. However, is important to provide adequate preoperative counselling, since anti-muscarinic therapy is less effective in women with de novo overactive bladder after mid-urethral sling placement [30].

The present study has several strengths including: (1) a clinical evaluation performed in all patients 20 years after TVT; (2) a highly homogeneous study population with the exclusion of women with mixed incontinence, DO and/or any other associated surgical procedure; (3) the subjective and objective outcomes available for the 1-, 5-, 10-, 15-, 17and 20 -year postoperative period. We acknowledge that the weaknesses of this study could be the limited sample size and the lack of data of pressure/flow study in all women with de novo OAB. However, we also emphasize that no larger investigations with a similar follow-up, evaluating both objective and subjective outcomes, are available in literature.

\section{Conclusion}

The present study, which is the first reporting a 20 -year follow-up, appears to demonstrate that TVT is associated with an excellent long-lasting objective and subjective cure rate, with a negligible rate of mesh-related complications. The dramatic impact of COVID 19 on the mortality rate of elderly women in the last 16 months has drastically reduced the number of eligible patients for future evaluations in our region.

Author's contribution Andrea Braga, PD: Protocol/Project development, Data collection, Statistical analysis, Manuscript writing.

Giorgio Caccia, Chief: Data collection, Critical revision.

Andrea Papadia, Full Professor: Critical revision.

Fabiana Castronovo, Resident: Data collection.

Stefano Salvatore, Associate Professor: Critical revision.

Chiara Scancarello, Resident: Data collection.

Marco Torella, Associate Professor: Critical revision.

Fabio Ghezzi, Full Professor: Critical revision.

Maurizio Serati, Associate Professor: Protocol/Project development, Data collection, Statistical analysis.

Funding Open access funding provided by Università della Svizzera italiana

\section{Declarations}

\section{Conflict of interest None.}

Open Access This article is licensed under a Creative Commons Attribution 4.0 International License, which permits use, sharing, adaptation, distribution and reproduction in any medium or format, as long as you give appropriate credit to the original author(s) and the source, provide a link to the Creative Commons licence, and indicate if changes were made. The images or other third party material in this article are included in the article's Creative Commons licence, unless indicated otherwise in a credit line to the material. If material is not included in the article's Creative Commons licence and your intended use is not permitted by statutory regulation or exceeds the permitted use, you will need to obtain permission directly from the copyright holder. To view a copy of this licence, visit http://creativecommons. org/licenses/by/4.0/.

\section{References}

1. Ulmsten U, Henriksson L, Johnson P, Varhos G. An ambulatory surgical procedure under local anesthesia for treatment of female urinary incontinence. Int Urogynecol J Pelvic Floor Dysfunct. 1996;7(2):81-5 discussion 85-6.

2. Braga A, Caccia G, Sorice $P$, et al. Tension-free vaginal tape for treatment of pure urodynamic stress urinary incontinence: efficacy and adverse effects at 17-year follow-up. BJU Int. 2018;122(1):113-7.

3. Serati M, Braga A, Caccia G, et al. TVT-O for treatment of pure urodynamic stress urinary incontinence incontinence: efficacy and adverse effects at 13-year follow-up. Neurourol Urodynam. 2017;36:192-7.

4. Braga A, Caccia G, Ruggeri G, et al. 3-year follow-up of tension-free vaginal tape-ABBREVO procedure for the treatment of pure urodynamic stress urinary incontinence: efficacy and adverse effects. Int Urogynecol J. 2020;31(4):739-44.

5. Considerations about Surgical Mesh for SUI I FDA. https:// www.fda.gov/medical-devices/urogynecologic-surgical-meshimplants/considerations-about-surgical-mesh-sui. Accessed 2 Aug 2020.

6. Berger AA, Tan-Kim J, Menefee A. The impact of the 2011 US Food and Drug Administration transvaginal mesh communication on utilization of synthetic mid-urethral sling procedures. Int Urogynecol J. 2020. https://doi.org/10.1007/s00192-020-04597-7.

7. Davila AGW, Su TH, for the FIGO Urogynecology and Pelvic Floor Committee. FIGO review of statements on use of synthetic mesh for pelvic organ prolapse and stress urinary incontinence. Int J Gynecol Obstet. 2019;147:147-55.

8. Fusco F, Abdel-Fattah M, Chapple CR, et al. Updated systematic review and Meta-analysis of the comparative data on Colposuspensions, Pubovaginal slings, and Midurethral tapes in the surgical treatment of female stress urinary incontinence. Eur Urol. 2017;S0302-2838(17):30334-2. https://doi.org/10.1016/j.eururo. 2017.04.026.

9. Chapple CR, Cruz F, Deffieux X, et al. Consensus statement of the European urology association and the European Urogynaecological association on the use of implanted materials for treating pelvic organ prolapse and stress urinary incontinence. Eur Urol. 2017;72:424-31.

10. Nilsson CG, Palva K, Aarnio R, et al. Seventeen years' follow-up of the tension-free vaginal tape procedure for female stress urinary incontinence. Int Urogynecol J. 2013;24(8):1265-9.

11. Tulokas S, Rahkola-Soisalo P, Gissler M, et al. Long-term reprocedure rate after mid-urethral slings for stress urinary incontinence. Int Urogynecol J. 2020;31(4):727-35.

12. Bump RC, Mattiasson A, Bo K, et al. The standardization of terminology of female pelvic organ prolapse and pelvic floor dysfunction. Am J Obstet Gynecol. 1996;175:10-7. 
13. Serati M, Salvatore S, Siesto G, et al. Urinary symptoms and urodynamic findings in women with pelvic organ prolapse: is there a correlation? Results of an artificial neural network analysis. Eur Urol. 2011;60:253-60.

14. Schafer W, Abrams P, Liao L, et al. Good urodynamic practices: uroflowmetry, filling cystometry, and pressure-flow studies. Neurourol Urodyn. 2002;21:261-74.

15. Haylen BT, de Ridder D, Freeman RM, et al. An international Urogynecological association (IUGA)/international continence society (ICS) joint report on the terminology for female pelvic floor dysfunction. Int Urogynecol J. 2010;21:5-26.

16. Avery K, Donovan J, Peters TJ, et al. A brief and robust measure for evaluating the symptoms and impact of urinary incontinence. Neurourol Urodyn. 2004;23:322-30.

17. Ghezzi F, Cromi A, Raio L, et al. Influence of anesthesia and hydrodissection on the complication rate after tension-free vaginal tape procedure. Eur J Obstet Gynecol Reprod Biol. 2005;118:95-8.

18. Serati M, Ghezzi F, Cattoni E, et al. Tension-free vaginal tape for the treatment of urodynamic stress incontinence: efficacy and adverse effects at 10-year follow-up. Eur Urol. 2012;61(5):93946. https://doi.org/10.1016/j.eururo.2012.01.038.

19. Yalcin I, Bump RC. Validation of two global impression questionnaires for incontinence. Am J Obstet Gynecol. 2003;189:98-101.

20. Campeau L, Tu LM, Lemieux MC, et al. A multicenter, prospective, randomized clinical trial comparing tension-free vaginal tape surgery and no treatment for the management of stress urinary incontinence in elderly women. Neurourol Urodyn. 2007;26:990-4.

21. Abdel-Fattah M, Ramsay I, Pringle S, et al. Evaluation of transobturator tension-free vaginal tapes in management of women with recurrent stress urinary incontinence. Urology. 2011;77:1070-5.

22. Rosmini F, Ferrigno L. Ethical aspects of epidemiological research. Istisan reports 15/44. Epidemiology and Public Health. National Institute of Health 2384-8936 (Italy). 2015.

23. European Scientific Committee on Emerging and Newly Identified Health Risks (SCENIHR). Final opinion on the safety of surgical meshes used in urogynecological surgery. Luxembourg: DG Health and Food Safety, Public Health, European Commission; 2015. http://ec.europa.eu/health/scientific_committees/emerging/ docs/scenihr_o_049.pdf.

24. Morling JR, McAllister DA, Agur W, et al. Adverse events after first, single, mesh and non-mesh surgical procedures for stress urinary incontinence and pelvic organ prolapse in Scotland, 19972016: a population-based cohort study. Lancet. 2017;389:629-40.

25. Scottish Government. The Scottish independent review of the use, safety and efficacy of transvaginal mesh implants in the treatment of stress urinary incontinence and pelvic organ prolapse in women: final report. Edinburgh: The Scottish Government www. gov.scot/Resource/0051/00515856.pdf. 2017.

26. Søgaard NB, Glavind K. Complications and re-operations after tension-free vaginal tape operation in women with stress urinary incontinence. Int Urogynecol J. 2021. https://doi.org/10.1007/ s00192-020-04402-5.

27. Ford AA, Rogerson L, Cody JD, et al. Mid-urethral sling operations for stress urinary incontinence in women. Cochrane Database Syst Rev. 2015;7:CD006375.

28. Chughtai B, Mao J, Matheny ME, et al. Long-term safety with sling mesh implants for stress. Incontinence J Urol. 2021;205:183-90.

29. MacCraith E, Forde JC, O'Brien FJ, et al. Contemporary trends for urological training and management of stress urinary incontinence in Ireland. Int Urogynecol J. 2021. https://doi.org/10.1007/ s00192-021-04887-8.

30. Serati M, Braga A, Sorice P, et al. Solifenacin in women with de novo overactive bladder after tension-free obturator vaginal tape-is it effective? J Urol. 2014;191(5):1322-6.

Publisher's note Springer Nature remains neutral with regard to jurisdictional claims in published maps and institutional affiliations. 